\title{
Generalizations of some classical theorems to D-normal operators on Hilbert spaces
}

M. Dana ${ }^{1 *}$ and R. Yousefi'

\section{"Correspondence:}

mansourdana44@gmail.com

${ }^{1}$ Faculty of Mathematics, University of Kurdistan, Sanandaj, Islamic

Republic of Iran

\section{Springer}

\begin{abstract}
We say that a Drazin invertible operator $T$ on Hilbert space is of class [DN] if $T^{D} T^{*}=T^{*} T^{D}$. The authors in (Oper. Matrices 12(2):465-487, 2018) studied several properties of this class. We prove the Fuglede-Putnam commutativity theorem for D-normal operators. Also, we show that $T$ has the Bishop property $(\beta)$. Finally, we generalize a very famous result on products of normal operators due to I. Kaplansky to D-normal matrices.
\end{abstract}

MSC: 47B15; 47B20; 15A09

Keywords: Drazin inverse; D-normal operator; Fuglede-Putnam theorem; Bishop property

\section{Introduction}

Let $\mathcal{H}$ be a complex Hilbert space. By $\mathcal{B}(\mathcal{H})$ we denote the space of all bounded linear operators on $\mathcal{H}$ and by $I=I_{\mathcal{H}}$ the identity operator. If $T \in \mathcal{B}(\mathcal{H})$, then $T^{*}$ denotes the adjoint of $T$. By $\mathcal{N}(T), \mathcal{R}(T)$, and $\sigma(T)$ we denote the null space, the range, and the spectrum of $T$, respectively. For convenience, we write $T-\lambda$ instead of $T-\lambda I$.

Property $(\beta)$ has been introduced by Bishop [4] and is defined as follows.

Definition 1.1 An operator $T \in \mathcal{B}(\mathcal{H})$ is said to have the Bishop property $(\beta)$ (shortly, property $(\beta))$ if for every open set $D$ of $\mathbb{C}$ and every sequence of analytic functions $f_{n}$ : $D \longrightarrow \mathcal{H}$ such that $(T-\mu) f_{n}(\mu) \longrightarrow 0$ uniformly on all compact subsets of $D$, then also $f_{n}(\mu) \longrightarrow 0$, again locally uniformly on $D$.

It is well known that every normal operator has property $(\beta)$. The study of operators satisfying property $(\beta)$ is of significant interest and is currently being done by a number of mathematicians around the world (see $[3,12])$.

Definition 1.2 Let $T \in \mathcal{B}(\mathcal{H})$. The operator $T$ is said to have the single-valued extension property at $\lambda \in \mathbb{C}$ (abbreviated SVEP at $\lambda$ ) if for every neighborhood $D$ of $\lambda$, the only analytic function $f: D \longrightarrow \mathcal{H}$ that satisfies the equation

$$
(T-\mu) f(\mu)=0
$$

(c) The Author(s) 2020. This article is licensed under a Creative Commons Attribution 4.0 International License, which permits use sharing, adaptation, distribution and reproduction in any medium or format, as long as you give appropriate credit to the original author(s) and the source, provide a link to the Creative Commons licence, and indicate if changes were made. The images or other third party material in this article are included in the article's Creative Commons licence, unless indicated otherwise in a credit line to the material. If material is not included in the article's Creative Commons licence and your intended use is not permitted by statutory regulation or exceeds the permitted use, you will need to obtain permission directly from the copyright holder. To view a copy of this licence, visit http://creativecommons.org/licenses/by/4.0/. 
is the constant function $f \equiv 0$.

The operator $T$ is said to have the SVEP if $T$ has the SVEP at every $\lambda \in \mathbb{C}$.

The quasinilpotent part and the analytic core of $(T-\lambda)$ are, respectively, defined by

$$
H_{0}(T-\lambda)=\left\{x \in \mathcal{H}: \lim _{n \rightarrow \infty}\left\|(T-\lambda)^{n} x\right\|^{\frac{1}{n}}=0\right\}
$$

and

$$
\begin{aligned}
K_{0}(T-\lambda)= & \left\{x \in \mathcal{H}: \text { there exist a sequence }\left(x_{n}\right) \subset \mathcal{H} \text { and a constant } \delta>0\right. \\
& \text { such that }(T-\lambda) x_{1}=x,(T-\lambda) x_{n+1}=x_{n}, \text { and }\left\|x_{n}\right\| \leq \delta^{n}\|x\| \\
& \text { for all } n \in \mathbb{N}\} .
\end{aligned}
$$

The subspace $C(T)$ in purely algebraic terms was introduced by Saphar [13].

Definition 1.3 Let $T$ be a linear operator on $\mathcal{H}$. The algebraic core $C(T)$ is is the greatest subspace $\mathcal{M}$ of $\mathcal{H}$ for which $T(\mathcal{M})=\mathcal{M}$.

For bounded linear operators, the Drazin inverse was introduced and studied by Caradus [6]. It is shown that the Drazin inverse is helpful in analyzing Markov chains, difference equation, differential equations, Cauchy problems, and iterative procedures [2, 5].

Definition 1.4 For $T \in \mathcal{B}(\mathcal{H})$, suppose that there exists an operator $T^{D} \in \mathcal{B}(\mathcal{H})$ satisfying the following three operator equations:

$$
T T^{D}=T^{D} T, \quad T^{D} T T^{D}=T^{D}, \quad T^{k+1} T^{D}=T^{k},
$$

where $k=\operatorname{ind}(T)$, the index of $T$, is the smallest nonnegative integer for which $\mathcal{R}\left(T^{k}\right)=$ $\mathcal{R}\left(T^{k+1}\right)$ and $\mathcal{N}\left(T^{k}\right)=\mathcal{N}\left(T^{k+1}\right)$. Then $T^{D}$ is called a Drazin inverse of $T$.

In particular, when $\operatorname{ind}(T)=1$, the operator $T^{D}$ is called the group inverse of $T$ and is denoted by $T^{\sharp}$. Clearly, ind $(T)=0$ if and only if $T$ is invertible, and in this case, $T^{D}=T^{-1}$.

Remark 1.5 Let $T$ be Drazin invertible.

1. The spectral idempotent $T^{\pi}$ of $T$ corresponding to $\{0\}$ is given by $T^{\pi}=I-T T^{D}$.

The operator matrix form of $T$ with respect to the space decomposition

$\mathcal{H}=\mathcal{N}\left(T^{\pi}\right) \oplus \mathcal{R}\left(T^{\pi}\right)$ is given by $T=T_{1} \oplus T_{2}$, where $T_{1}$ is invertible, and $T_{2}$ is nilpotent.

2. $\quad H_{0}(T)=\mathcal{R}\left(T^{\pi}\right)=\mathcal{N}\left(T^{D}\right)=\mathcal{N}\left(T^{k}\right)$

- $K_{0}(T)=\mathcal{N}\left(T^{\pi}\right)=\mathcal{R}\left(T^{D}\right)=\mathcal{R}\left(T^{k}\right)$, where $k=\operatorname{ind}(T)$.

For $T \in \mathcal{B}(\mathcal{H})$, it is well known that the Drazin inverse $T^{D}$ of $T$ is unique if it exists, and then $\left(T^{*}\right)^{D}=\left(T^{D}\right)^{*}$.

Lemma 1.6 ([5]) Let $S, T \in \mathcal{B}(\mathcal{H})$ be Drazin invertible. Then 
(i) ST is Drazin invertible if and only if TS is Drazin invertible, $\operatorname{ind}(S T) \leq \operatorname{ind}(T S)+1$, and $(S T)^{D}=S\left[(T S)^{D}\right]^{2} T$.

(ii) If $S$ is idempotent, then $S^{D}=S$.

(iii) If $S T=T S$, then $(S T)^{D}=T^{D} S^{D}=S^{D} T^{D}, S^{D} T=T S^{D}$, and $S T^{D}=T^{D} S$.

Definition $1.7([7])$ Let $T \in \mathcal{B}(\mathcal{H})$ be Drazin invertible. $T$ is called a $D$-normal operator if

$$
T^{D} T^{*}=T^{*} T^{D}
$$

The class of all $D$-normal operators is denoted by $[D N]$.

Proposition 1.8 Let $T \in \mathcal{B}(\mathcal{H})$ be Drazin invertible. Then $T$ is $D$-normal if and only if $T^{D}$ is normal.

Proof Let $T$ be $D$-normal. Then $T^{D} T^{*}=T^{*} T^{D}$ and, by Lemma 1.6(3), $T^{D}\left(T^{*}\right)^{D}=$ $\left(T^{*}\right)^{D} T^{D}$. Since $\left(T^{*}\right)^{D}=\left(T^{D}\right)^{*}, T^{D}$ is normal. Now let $T^{D}$ be normal. Since $T^{D} T=T T^{D}$, by the Fuglede theorem, $T^{D} T^{*}=T^{*} T^{D}$. Therefore $T$ is $D$-normal.

$D$-normal operators were introduced and studied by Dana and Yousefi [7]. The authors in $[8,9]$ studied several properties of this class.

\section{Fuglede-Putnam theorem for $D$-normal operators}

The Fuglede-Putnam theorem is a very useful tool when dealing with products (and even sums) involving normal operators. As an application of this theorem, we can name Kaplansky theorem [10]. Many mathematicians attempt to extend this theorem to nonnormal operators (see [14]).

The Hilbert-Schmidt operators in $\mathcal{H}$ form an ideal $\mathbb{H}$ in the algebra $\mathcal{B}(\mathcal{H})$ of all operators in $\mathcal{H}$. The ideal $\mathbb{H}$ itself is a Hilbert space with inner product

$$
\langle X, Y\rangle=\sum\left\langle X e_{i}, Y e_{i}\right\rangle=\operatorname{tr}\left(Y^{*} X\right)=\operatorname{tr}\left(X Y^{*}\right)
$$

where $\left\{e_{i}\right\}$ is any orthonormal basis of $\mathcal{H}$. For each pair of operators $S, T \in \mathcal{B}(\mathcal{H})$, there is an operator $\Gamma$ defined on $\mathcal{B}(\mathcal{H})$ by the formula $\Gamma X=S X T$ as in [3]. The adjoint and the Drazin inverse of $\Gamma$ are given by the formulas

$$
\Gamma^{*} X=S^{*} X T^{*} \text { and } \Gamma^{D} X=S^{D} X T^{D}
$$

We say that normal operators $S, T$ satisfy the Fuglede-Putnam theorem if $S X=X T$ implies $S^{*} X=X T^{*}$. The aim of this section is to show that if $S, T$ are of class $[D N]$ and $T$ is invertible, then for a Hilbert-Schmidt operator $X$,

$$
S X=X T \text { implies } S^{*} X=X T^{*} \text {. }
$$

Theorem 2.1 Let $S, T, X \in \mathcal{B}(\mathcal{H})$ be such that $S$ and $T$ are Drazin invertible. If $S X=X T$, then $S^{D} X=X T^{D}$. 
Proof There exists a scalar polynomial $g$ such that $(S \oplus T)^{D}=g(S \oplus T)$ [5]. This implies that $S^{D}=g(S)$ and $T^{D}=g(T)$. Hence $S^{D} X=g(S) X=X g(T)=X T^{D}$.

Lemma 2.2 If $S, T \in[D N]$, then the operator $\Gamma$ is of class $[D N]$.

Proof By hypothesis, $S^{D} S^{*}=S^{*} S^{D}$ and $T^{D} T^{*}=T^{*} T^{D}$. For any pair $S, T \in \mathcal{B}(\mathcal{H})$,

$$
\begin{aligned}
\left(\Gamma^{*} \Gamma^{D}-\Gamma^{D} \Gamma^{*}\right) X & =\Gamma^{*} \Gamma^{D} X-\Gamma^{D} \Gamma^{*} X \\
& =\Gamma^{*}\left(S^{D} X T^{D}\right)-\Gamma^{D}\left(S^{*} X T^{*}\right) \\
& =S^{*}\left(S^{D} X T^{D}\right) T^{*}-S^{D}\left(S^{*} X T^{*}\right) T^{D} \\
& =0,
\end{aligned}
$$

which implies that $\Gamma$ is of class $[D N]$.

Theorem 2.3 Let $S, T \in[D N] n r$ such that $T$ is invertible, and let $X$ be a Hilbert-Schmidt operator. If $S X=X T$, then $S^{*} X=X T^{*}$.

Proof Let $\Gamma$ be the Hilbert-Schmidt operator defined by $\Gamma Y=S Y T^{-1}$, where $Y \in \mathcal{B}(\mathcal{H})$. Since $S, T$ are of class $[D N]$, by Lemma 2.2, $\Gamma$ is of class $[D N]$. The hypothesis $S X=X T$ implies that $\Gamma X=X$ and $\Gamma^{D} X=X$ and also

$$
\begin{aligned}
\left\|\Gamma^{*} X\right\|^{2} & =\left\langle\Gamma^{*} X, \Gamma^{*} X\right\rangle \\
& =\left\langle\Gamma^{*}\left(\Gamma^{D}\right)^{2} X, \Gamma^{*}\left(\Gamma^{D}\right)^{2} X\right\rangle \\
& =\left\langle\Gamma\left(\Gamma^{D}\right)^{2^{*}} \Gamma^{*}\left(\Gamma^{D}\right)^{2} X, X\right\rangle \\
& =\left\langle\Gamma^{D} X, \Gamma^{D} X\right\rangle \\
& =\|X\|^{2} .
\end{aligned}
$$

On the other hand,

$$
\begin{aligned}
\left\langle\Gamma^{*} X, X\right\rangle & =\left\langle\Gamma^{*} X,\left(\Gamma^{D}\right)^{2} X\right\rangle \\
& =\left\langle\left(\Gamma^{D}\right)^{2^{*}} \Gamma^{*} X, X\right\rangle \\
& =\left\langle\Gamma^{D^{*}} X, X\right\rangle \\
& =\left\langle X, \Gamma^{D} X\right\rangle \\
& =\langle X, X\rangle .
\end{aligned}
$$

So we have

$$
\begin{aligned}
\left\|\Gamma^{*} X-X\right\|^{2} & =\left\langle\Gamma^{*} X-X, \Gamma^{*} X-X\right\rangle \\
& =\left\langle\Gamma^{*} X, \Gamma^{*} X\right\rangle-\left\langle\Gamma^{*} X, X\right\rangle-\left\langle X, \Gamma^{*} X\right\rangle+\langle X, X\rangle \\
& =\left\|\Gamma^{*} X\right\|^{2}-\left\langle\Gamma^{*} X, X\right\rangle-\left\langle X, \Gamma^{*} X\right\rangle+\|X\|^{2} \\
& =0 .
\end{aligned}
$$

Therefore $\Gamma^{*} X=X$, and hence $S^{*} X=X T^{*}$. 
Here we give an example that if $X \in \mathcal{B}(\mathcal{H})$ and $S, T \in[D N]$ satisfy $S X=X T$, then we cannot get $S^{*} X=X T^{*}$. Just consider the operator $S=X=\left(\begin{array}{ll}0 & 0 \\ 1 & 0\end{array}\right)$ and $T=0$. Then $S X=X T$, but $S^{*} X=\left(\begin{array}{ll}1 & 0 \\ 0 & 0\end{array}\right)$ and $X T^{*}=\left(\begin{array}{ll}0 & 0 \\ 0 & 0\end{array}\right)$.

\section{Bishop property for $D$-normal operators}

We start this section with the matrix representation for $T \in[D N]$.

Lemma 3.1 If $T \in[D N]$, then $\mathcal{R}\left(T^{D}\right)$ reduces $T$.

Proof Since $T \in[D N], T^{D} T^{*}=T^{*} T^{D}$. Obviously, $\mathcal{R}\left(T^{D}\right)$ is invariant under $T$. We will show that $\mathcal{R}\left(T^{D}\right)$ is invariant under $T^{*}$. Let $x \in \mathcal{R}\left(T^{D}\right)$. Then $x=T^{D} y$ for some $y \in \mathcal{H}$, and $T^{*} x=T^{*} T^{D} y=T^{D} T^{*} y \in \mathcal{R}\left(T^{D}\right)$. Thus $\mathcal{R}\left(T^{D}\right)$ is invariant under $T^{*}$, and $\mathcal{R}\left(T^{D}\right)$ reduces $T$.

Theorem 3.2 If $T$ is of class [DN], then $T$ has the following matrix representation: $T=$ $\left(\begin{array}{cc}T_{1} & 0 \\ 0 & T_{2}\end{array}\right)$ on $\mathcal{H}=\mathcal{R}\left(T^{D}\right) \oplus \mathcal{N}\left(T^{D}\right)$, where $T_{1}=\left.T\right|_{\mathcal{R}\left(T^{D}\right)}$ is also of class $[N]$, and $T_{2}$ is a nilpotent operator with nilpotency $\operatorname{ind}(T)$. Furthermore, $\sigma(T)=\sigma\left(T_{1}\right) \cup\{0\}$.

Proof By Lemma 3.1, $\mathcal{R}\left(T^{D}\right)$ reduces $T$. Hence $T$ has the matrix representation $T=$ $\left(\begin{array}{cc}T_{1} & 0 \\ 0 & T_{2}\end{array}\right)$ on $\mathcal{H}=\mathcal{R}\left(T^{D}\right) \oplus \mathcal{N}\left(T^{* D}\right)$. Note that since $T \in[D N], \mathcal{N}\left(T^{D}\right)=\mathcal{N}\left(T^{* D}\right)$. Let $P$ be the orthogonal projection onto $\mathcal{R}\left(T^{D}\right)$. Then

$$
\left(\begin{array}{cc}
T_{1} & 0 \\
0 & 0
\end{array}\right)=T P=P T=P T P .
$$

Hence

$$
P\left(T^{D} T^{*}\right) P=\left(\begin{array}{cc}
T_{1}^{D} T_{1}^{*} & 0 \\
0 & 0
\end{array}\right)
$$

and

$$
P\left(T^{*} T^{D}\right) P=\left(\begin{array}{cc}
T_{1}^{*} T_{1}^{D} & 0 \\
0 & 0
\end{array}\right)
$$

Since $T \in[D N], P\left(T^{*} T^{D}\right) P=P\left(T^{D} T^{*}\right) P$, implying $T_{1}^{*} T_{1}^{D}=T_{1}^{D} T_{1}^{*}$. Hence $T_{1} \in[D N]$. On the other hand, by Remark 1.5, $T_{1}$ is invertible. So $T_{1} \in[N]$.

$$
\begin{aligned}
& \text { For any } z=\left(\begin{array}{l}
z_{1} \\
z_{2}
\end{array}\right) \in \mathcal{H}, \\
& \qquad \begin{aligned}
\left\langle T_{2}^{D} z_{2}, z_{2}\right\rangle & =\left\langle T^{D}(I-P) z,(I-P) z\right\rangle \\
& =\left\langle(I-P) z,\left(T^{D}\right)^{*}(I-P) z\right\rangle \\
& =0 .
\end{aligned}
\end{aligned}
$$

Therefore $T_{2}^{D}=0$. Then $T_{2}$ is a nilpotent operator. Since $\mathcal{R}\left(T^{D}\right)$ reduces $T, \sigma(T)=\sigma\left(T_{1}\right) \cup$ $\sigma\left(T_{2}\right)=\sigma\left(T_{1}\right) \cup\{0\}$.

Theorem 3.3 If $T \in[D N]$, then $T$ has property $(\beta)$. 
Proof If $D \subset \mathbb{C}$ is an open neighborhood of $\lambda \in \mathbb{C}$ and $f_{m}(m=1,2, \ldots)$ are vector-valued analytic functions on $D$ such that $(T-\mu) f_{m}(\mu) \longrightarrow 0$ uniformly on every compact subset of $D$, then we decompose $\mathcal{H}$ as $\mathcal{H}=\mathcal{R}\left(T^{D}\right) \oplus \mathcal{N}\left(T^{D}\right)$, and by Theorem 3.2 $T=\left(\begin{array}{cc}T_{1} & 0 \\ 0 & T_{2}\end{array}\right)$ where $T_{1} \in[N]$, and $T_{2}$ is a nilpotent operator with nilpotency $\operatorname{ind}(T)$. The convergence $(T-\mu) f_{m}(\mu) \longrightarrow 0$ implies

$$
\left(\begin{array}{cc}
T_{1}-\mu & 0 \\
0 & T_{2}-\mu
\end{array}\right)\left(\begin{array}{l}
f_{m_{1}}(\mu) \\
f_{m_{2}}(\mu)
\end{array}\right)=\left(\begin{array}{l}
\left(T_{1}-\mu\right) f_{m_{1}}(\mu) \\
\left(T_{2}-\mu\right) f_{m_{2}}(\mu)
\end{array}\right)
$$

Since $T_{2}$ is nilpotent, it has property $(\beta)$, and therefore $f_{m_{2}}(\mu) \longrightarrow 0$. Also, since $T_{1}$ is normal, it has property $(\beta)$. So by Theorem 3.39 in [11], $T$ has property $(\beta)$.

From the theorem we immediately have the following:

Corollary 3.4 If $T \in[D N]$, then $T$ has the SVEP.

The following example shows that for a $D$-normal operator $T$, the corresponding eigenspaces need not be reducing subspaces of $T$.

Example $3.5 T=\left(\begin{array}{ll}0 & 1 \\ 0 & 0\end{array}\right)$. Clearly, $T$ is a $D$-normal operator, and the eigenspace of $T$ is $\left(\begin{array}{l}x \\ 0\end{array}\right)$, but it is not a reducing subspace of $T$.

Theorem 3.6 Suppose that $T \in[D N]$. Then $C\left(T^{D}\right)$ is invariant under $T^{*}$.

Proof By the definition of algebraic core of $T^{D}, T^{D}\left(C\left(T^{D}\right)\right)=C\left(T^{D}\right)$. Since $T \in[D N]$, $T^{*} T^{D}=T^{D} T^{*}$. So we have $T^{*} T^{D} C\left(T^{D}\right)=T^{D} T^{*} C\left(T^{D}\right)$. This implies $T^{*} C\left(T^{D}\right)=T^{D} T^{*} \times$ $C\left(T^{D}\right)$. Now, since $C\left(T^{D}\right)$ is the greatest subspace satisfying $T^{D}\left(C\left(T^{D}\right)\right)=C\left(T^{D}\right)$, we have $T^{*} C\left(T^{D}\right) \subseteq C\left(T^{D}\right)$. Thus $C\left(T^{D}\right)$ is invariant under $T^{*}$.

Theorem 3.7 If $T \in[D N]$, then the following properties hold:

1. $H_{0}\left(T^{D}-\lambda\right)$ is a reducing subspace of $T$.

2. $x \in H_{0}(T)$ if and only if $T^{*} x \in H_{0}(T)$.

3. $H_{0}\left(T^{D}-\lambda\right)=\mathcal{N}\left(T^{D}-\lambda\right)=\mathcal{N}\left(T^{D}-\lambda\right)^{*}$. In particular, $H_{0}(T)=\mathcal{N}\left(T^{D}\right)=\mathcal{N}\left(\left(T^{D}\right)^{*}\right)$.

4. If $\mathcal{M}$ is an invariant subspace of $T$ and $T_{1}=\left.T\right|_{\mathcal{M}}$ on $\mathcal{H}=\mathcal{M} \oplus \mathcal{M}^{\perp}$, then

$$
H_{0}\left(T_{1}^{D}-\lambda\right)=\mathcal{N}\left(T_{1}^{D}-\lambda\right)=\mathcal{N}\left(T_{1}^{D}-\lambda\right)^{*}
$$

Proof 1. Since $T \in[D N],\left(T^{D}-\lambda\right) T^{*}=T^{*}\left(T^{D}-\lambda\right)$, and hence for $x \in H_{0}\left(T^{D}-\lambda\right)$, we have

$$
\begin{aligned}
\lim _{n \rightarrow \infty}\left\|\left(T^{D}-\lambda\right)^{n} T^{*} x\right\|^{\frac{1}{n}} & =\lim _{n \rightarrow \infty}\left\|T^{*}\left(T^{D}-\lambda\right)^{n} x\right\|^{\frac{1}{n}} \\
& \leq \lim _{n \rightarrow \infty}\left\|T^{*}\right\|^{\frac{1}{n}}\left\|\left(T^{D}-\lambda\right)^{n} x\right\|^{\frac{1}{n}} \\
& =0 .
\end{aligned}
$$

Hence $T^{*} x \in H_{0}\left(T^{D}-\lambda\right)$. It is easy to see that $T x \in H_{0}\left(T^{D}-\lambda\right)$.

2. We have $H_{0}(T)=\mathcal{N}\left(T^{D}\right)$. On the other hand, $\left(T^{D}\right)^{D}=T^{2} T^{D}$. It is clear that $\mathcal{N}\left(T^{D}\right)=$ $\mathcal{N}\left(T^{2} T^{D}\right)$. So, $H_{0}(T)=H_{0}\left(T^{D}\right)$. 
If $x \in H_{0}(T)=H_{0}\left(T^{D}\right)$, then we easily get that $T^{*} x \in H_{0}(T)$. To prove the converse, let $T^{*} x \in H_{0}(T)$. For every $n>1$, we have

$$
\begin{aligned}
\left\|\left(T^{D}\right)^{n} T^{*} x\right\|^{2} & =\left\langle\left(T^{D}\right)^{n} T^{*} x,\left(T^{D}\right)^{n} T^{*} x\right\rangle \\
& =\left\langle T^{*}\left(T^{D}\right)^{n} x, T^{*}\left(T^{D}\right)^{n} x\right\rangle \\
& =\left\langle\left(T^{* D}\right)^{n} T T^{*}\left(T^{D}\right)^{n} x, x\right\rangle \\
& =\left\langle T\left(T^{* D}\right)^{n} T^{*}\left(T^{D}\right)^{n} x, x\right\rangle \\
& =\left\langle T\left(T^{D}\right)^{n}\left(T^{* D}\right)^{n} T^{*} x, x\right\rangle \\
& =\left\langle\left(T^{* D}\right)^{n-1} x,\left(T^{* D}\right)^{n-1} x\right\rangle \\
& =\left\|\left(T^{* D}\right)^{n-1} x\right\|^{2} \\
& =\left\|\left(T^{D}\right)^{n-1} x\right\|^{2} .
\end{aligned}
$$

So, for every $n>1$,

$$
\left\|\left(T^{D}\right)^{n} T^{*} x\right\|^{2}=\left\|\left(T^{D}\right)^{n-1} x\right\|^{2},
$$

and for $n=1$,

$$
\left\|T^{D} T^{*} x\right\|^{2}=\left\|T T^{D} x\right\|^{2}
$$

According to (3.1),

$$
\lim _{n \rightarrow \infty}\left\|\left(T^{D}\right)^{n-1} x\right\|^{\frac{1}{n-1}}=\lim _{n \rightarrow \infty}\left(\left\|\left(T^{D}\right)^{n} T^{*} x\right\|^{\frac{1}{n}}\right)^{\frac{n}{n-1}}=0 .
$$

Thus $x \in H_{0}\left(T^{D}\right)=H_{0}(T)$.

3. Notice that for a totally paranormal operator $T, H_{0}(T-\lambda)=\mathcal{N}(T-\lambda)$ for every $\lambda \in \mathbb{C}$ [1]. The class of totally paranormal operators includes the class of hyponormal operators and hence normal operators. In view of normality $T^{D}$, we have

$$
H_{0}\left(T^{D}-\lambda\right)=\mathcal{N}\left(T^{D}-\lambda\right)=\mathcal{N}\left(T^{D}-\lambda\right)^{*}
$$

For $\lambda=0, H_{0}\left(T^{D}\right)=\mathcal{N}\left(T^{D}\right)=\mathcal{N}\left(T^{D}\right)^{*}$.

3. By Proposition 2.6 of [7], $T_{1}^{D}=\left.T^{D}\right|_{\mathcal{M}}$ is hyponormal, and hence $H_{0}\left(T_{1}^{D}-\lambda\right)=$ $\mathcal{N}\left(T_{1}^{D}-\lambda\right)=\mathcal{N}\left(T_{1}^{D}-\lambda\right)^{*}$.

\section{Generalization of Kaplansky theorem for $D$-normal matrices}

Let $\mathcal{M}_{n}(\mathbb{C})$ be the set of $n \times n$ complex matrices. In this section, we are mainly interested in generalizing the following famous result on products of normal operators, due to I. Kaplansky, to $D$-normal matrices.

Theorem 4.1 ([10]) Let $A$ and $B$ be two bounded operators on a Hilbert space such that $A B$ and $A$ are normal. Then $B$ commutes with $A A^{*}$ iff $B A$ is normal. 
Proposition 4.2 Let $A, B \in \mathcal{M}_{n}(\mathbb{C})$ be such that $A B$ is $D$-normal. Then

$$
A^{*} A B=B A A^{*} \quad \Longrightarrow \quad B A \text { is D-normal. }
$$

Proof Let $A=U P$, where $P$ is positive, and $U$ is unitary. Note that there exists a positive semidefinite $K \in \mathcal{M}_{n}(\mathbb{C})$ such that $A=K U$. We obtain

$$
\begin{aligned}
P^{2} B & =A^{*} A B \\
& =(B A) A^{*} \\
& =B K^{2} .
\end{aligned}
$$

Hence, since $P$ and $K$ are positive semidefinite, $P B=B K$. Then $P B U=B K U$. So $P B U=$ BUP. Thus

$$
U^{*} A B U=U^{*} U P B U=P B U=B A .
$$

Hence $B A$ is unitary equivalent to a $D$-normal operator, and thus by [7, Proposition 2.6], it is $D$-normal itself.

Remark 4.3 Using a similar method as in Proposition 4.2, we can show that for $A, B \in$ $\mathcal{M}_{n}(\mathbb{C})$, by the Kaplansky theorem the condition that $A$ is normal is superfluous.

Proposition 4.4 Let $A, B \in \mathcal{M}_{n}(\mathbb{C})$ be such that $A B$ is D-normal. Then

$$
A^{*}(A B)^{D}=(B A)^{D} A^{*} \quad \Longleftrightarrow \quad B A \text { is D-normal. }
$$

Proof Let $A B$ and $B A$ be $D$-normal matrices. Then by Lemma 1.6(i)

$$
\begin{aligned}
A(B A)^{D} & =A B\left((A B)^{2}\right)^{D} A \\
& =(A B)^{D} A .
\end{aligned}
$$

Hence, by the Fuglede-Putnam theorem,

$$
A\left((B A)^{D}\right)^{*}=\left((A B)^{D}\right)^{*} A
$$

So,

$$
A^{*} A\left((B A)^{D}\right)^{2} B=B\left((A B)^{D}\right)^{2} A A^{*} .
$$

Hence

$$
A^{*}(A B)^{D}=(B A)^{D} A^{*}
$$

Conversely, if $A^{*}(A B)^{D}=(B A)^{D} A^{*}$, then $A^{*} A\left((B A)^{D}\right)^{2} B=B\left((A B)^{D}\right)^{2} A A^{*}$. Let $A=U P$, where $P$ is positive, and $U$ is unitary. Note that there exists a positive semidefinite $K \in$ 
$\mathcal{M}_{n}(\mathbb{C})$ such that $A=K U$. So $P^{2}\left((B A)^{D}\right)^{2} B=B\left((A B)^{D}\right)^{2} K^{2}$. Hence, since $P$ and $K$ are positive semidefinite, $P\left((B A)^{D}\right)^{2} B=B\left((A B)^{D}\right)^{2} K$. So we have

$$
P\left((B A)^{D}\right)^{2} B U=B\left((A B)^{D}\right)^{2} K U .
$$

Now

$$
\begin{aligned}
U^{*}(A B)^{D} U & =U^{*} A\left((B A)^{D}\right)^{2} B U \quad \text { (by Lemma 1.6) } \\
& =U^{*} U P\left((B A)^{D}\right)^{2} B U \\
& =B\left((A B)^{D}\right)^{2} K U \quad(\text { by }(4.1)) \\
& =B\left((A B)^{D}\right)^{2} A \\
& =(B A)^{D} .
\end{aligned}
$$

Hence $(B A)^{D}$ is unitary equivalent to a normal operator and thus is normal itself.

\section{Acknowledgements}

The authors would like to thank the anonymous referees for careful reading and helpful comments and suggestions for improving this paper.

\section{Funding}

This research received no specific grant form any funding agency in the public, commercial, or not-for-profit sectors.

\section{Availability of data and materials}

All data generated or analyzed during this study are included in this paper.

\section{Competing interests}

The authors declare that they have no competing interests.

\section{Authors' contributions}

All authors equally contributed to each part of this work. All authors read and approved the final manuscript.

\section{Publisher's Note}

Springer Nature remains neutral with regard to jurisdictional claims in published maps and institutional affiliations.

Received: 14 May 2019 Accepted: 3 April 2020 Published online: 15 April 2020

\section{References}

1. Aiena, P., Biondi, M.T., Villafane, F.: Weyl's theorems and Kato spectrum. Divulg. Mat. 15, 123-142 (2007)

2. Ben-Israel, A., Greville, T.N.E.: Generalized Inverses: Theory and Applications, 2nd edn. Springer, New York (2003)

3. Berberian, S.K.: Extensions of a theorem of Fuglede and Putnam. Proc. Am. Math. Soc. 71, 113-114 (1978)

4. Bishop, E.: A duality theorem for an arbitrary operator. Pac. J. Math. 9, 379-397 (1959)

5. Campbell, S.L., Meyer, C.D.: Generalized Inverse of Linear Transformations. Pitman, London (1979). Dover, New York (1991)

6. Caradus, S.R.: Operator Theory of the Generalized Inverse. Science Press, New York (2004)

7. Dana, M., Yousefi, R.: On the classes of $D$-normal operators and $D$-quasi-normal operators on Hilbert space. Oper. Matrices 12(2), 465-487 (2018)

8. Dana, M., Yousefi, R.: Some results on the classes of D-normal operators and n-power D-normal operators. Results Math. 74, Article ID 24 (2019)

9. Dana, M., Yousefi, R.: On a new class of generalized normal operators. Complex Anal. Oper. Theory 13, 3569-3578 (2019)

10. Kaplansky, I.: Products of normal operators. Duke Math. J. 20(2), 257-260 (1953)

11. Laursen, K.B., Neumann, M.M.: An Introduction to Local Spectral Theory. London Mathematical Society Monographs New Series, vol. 20 (2000)

12. Mary, J.S.I., Vijayalakshmi, P.: Fuglede-Putnam theorem and quasi-nilpotent part of $n$-power operators. Tamkang J. Math. 46(2), 151-165 (2015)

13. Saphar, P.: Contribution à l'étude des applications linéaires dans un espace de Banach. Bull. Soc. Math. Fr. 92, 363-384 (1964)

14. Yuan, J.T., Wang, C.H.: Fuglede-Putnam type theorems for $(p, k)$-quasihyponormal operators via hyponormal operators. J. Inequal. Appl. 2019, Article ID 122 (2019) 